\title{
Real-World Postmarketing Study of the Impact of Adalimumab Treatment on Work Productivity and Activity Impairment in Patients with Psoriatic Arthritis
}

\author{
Hidemi Nakagawa · Yoshiya Tanaka - Shigetoshi Sano • \\ Hideto Kameda - Atsuo Taniguchi · Tomoko Kashiwagi • \\ Takeshi Kawaberi · Junko Kimura · Akimichi Morita
}

Received: October 29, 2018 / Published online: January 19, 2019

(C) The Author(s) 2019

\section{ABSTRACT}

Introduction: This study investigated the effectiveness of adalimumab treatment in improving Work Productivity and Activity Impairment (WPAI) in patients with psoriatic arthritis (PsA) in real-world settings in Japan.

Methods: This 24-week, single-arm, postmarketing surveillance study (2014-2017), con-

Hidemi Nakagawa and Yoshiya Tanaka contributed equally as first authors.

Enhanced digital features To view enhanced digital features for this article go to https://doi.org/10.6084/ m9.figshare.7484744.

Electronic supplementary material The online version of this article (https://doi.org/10.1007/s12325018-0866-y) contains supplementary material, which is available to authorized users.

H. Nakagawa

Department of Dermatology, The Jikei University

School of Medicine, Tokyo, Japan

Y. Tanaka

The First Department of Internal Medicine, School of Medicine, University of Occupational and Environmental Health Japan, Kitakyushu, Japan

S. Sano

Department of Dermatology, Kochi Medical School, Kochi University, Kochi, Japan

H. Kameda

Division of Rheumatology, Toho University, Tokyo, Japan ducted at 75 centers in Japan, enrolled adalimumab-naïve patients (paid workers, including part-time) meeting ClASsification criteria for Psoriatic ARthritis (CASPAR). The primary endpoint was improvement in overall work impairment (OWI) scores from baseline to week 24. Secondary endpoints included changes in WPAI-PsA (OWI, absenteeism, presenteeism, and activity impairment), Psoriasis Area and Severity Index (PASI), psoriatic arthritis screening and evaluation (PASE) scores, Disease Activity Scores in 28 joints using C-reactive protein (DAS28[CRP]), Bath Ankylosing Spondylitis Disease Activity Index (BASDAI) scores, Health Assessment Questionnaire-Disability Index (HAQ-DI) scores, and PASI75/90 and American College of Rheumatology (ACR) 20/50/70 rates.

Results: In the effectiveness population $(n=106$; $72.6 \%$ men; mean \pm standard deviation $[S D]$ age,

\section{A. Taniguchi}

Institute of Rheumatology, Tokyo Women's Medical University, Tokyo, Japan

T. Kashiwagi · T. Kawaberi · J. Kimura ( $₫)$

AbbVie GK, 3-5-27, Mita, Minato-ku, Tokyo

108-6302, Japan

e-mail: junko.kimura@abbvie.com

\section{A. Morita}

Department of Geriatric and Environmental

Dermatology, Nagoya City University Graduate

School of Medical Sciences, Nagoya, Japan 
$49.3 \pm 10.7$ years), OWI scores significantly improved (mean \pm SD change, $-25.2 \pm 35.3$; $p<0.0001$ ) from baseline to week 24. Other WPAI domain scores also improved significantly. Changes in OWI were significantly correlated $(p<0.0001)$ with PASE $(r=0.6284)$, DAS28(CRP) $(r=0.6059)$, BASDAI $(r=0.7281)$, and HAQ-DI $(r=0.6161)$ scores and were significantly influenced by previous nonsteroidal anti-inflammatory drug use $(p=0.0142)$, and baseline PASE $(p=0.0098)$, DAS28(CRP) $(p=0.0026)$, HAQ-DI $(p=0.0004)$, and BASDAI $(p<0.0001)$ scores. At the last evaluation, rate (95\% confidence interval) of PASI 75 and $90(n=100)$ was $58.0 \%(47.7-67.8)$ and $39.0 \%$ (29.4-49.3), respectively, and that of ACR 20, 50, and $70 \quad(n=58)$ was $86.2 \%$ (74.6-93.9), $70.7 \% \quad(57.3-81.9)$, and $53.4 \%$ (39.9-66.7), respectively. No new safety signals were observed in the safety population $(n=148)$. Conclusion: Adalimumab treatment improved WPAI in patients with PsA. Improvements in OWI and joint symptoms were significantly associated.

Trial Registration Number: ClinicalTrials.gov identifier: NCT02414633.

Funding: AbbVie GK and Eisai Co., Ltd.

Keywords: Adalimumab; Japan; Postmarketing surveillance study; Psoriasis; Psoriatic arthritis; Rheumatology; TNF inhibitor; Work disability; Work productivity; Activity impairment

\section{INTRODUCTION}

Psoriatic arthritis (PsA) is a chronic, debilitating, inflammatory disease that substantially affects work disability (absenteeism and presenteeism), activity impairment (AI) and productivity, and the employment status of patients [1-3]. The exact global prevalence of PsA in the general population is unknown, but is estimated to be $0.3 \%-1.0 \%$ [4]. In Japan, the mean prevalence of PsA among patients with psoriasis was estimated to be 3.3\% between 2002 and 2008 [5, 6]. Based on annual surveys by the Japanese Society for Psoriasis Research, the prevalence of PsA has risen over the years, and was 15.3\% in 2016 [7]. PsA is almost twice as common in men as women, with a mean age of onset in the late 40 s
[8], indicating a working cohort. Similar to the rest of the world [3], PsA in Japanese patients is significantly associated with overall work impairment (OWI) and AI [9].

In the European League Against Rheumatism (EULAR) updated 2015 guidelines, conventional synthetic disease-modifying antirheumatic drugs (cs-DMARDs) are recommended for the initial management of patients with PsA after failure of nonsteroidal anti-inflammatory drugs (NSAIDs) and local therapy for active disease. In cases of inadequate response to cs-DMARDs, treatment with biologics, usually a tumor necrosis factor (TNF)- $\alpha$ inhibitor, is recommended [10]. According to the 2013 version of the Japanese guidance for the use of biologics for psoriasis, biologics are recommended in the early stages of PsA to prevent irreversible joint destruction [11].

The TNF inhibitor adalimumab (HUMIRA ${ }^{\circledR}$; AbbVie Inc., North Chicago, IL, USA) was one of the first biologic agents approved in Japan for the treatment of patients with psoriasis, including PsA [12]. For the treatment of PsA, adalimumab is administered subcutaneously at an initial loading dose of $80 \mathrm{mg}$, followed by 40-mg doses every other week (q2w) starting from the second week. The dosage may be increased to $80 \mathrm{mg} \mathrm{q} 2 \mathrm{w}$ when the response to the 40-mg dose is inadequate [13]. In a phase $2 / 3$ trial involving Japanese patients with moderate to severe chronic plaque psoriasis, adalimumab treatment resulted in significantly greater improvements in Psoriasis Area and Severity Index (PASI) 75 scores at three different dosing regimens $(40 \mathrm{mg}$ q2 $\mathrm{w}, 40 \mathrm{mg}$ q2w with an 80-mg loading dose, and $80 \mathrm{mg}$ q2w) than placebo $(57.9 \%, 62.8 \%$, and $81.0 \%$, respectively, vs $4.3 \%$ ) [14]. In a subgroup analysis of 16-week data from the Randomized controlled EValuation of adalimumab Every other week dosing in moderate to severe psoriasis triAL (REVEAL), which included employed patients with moderate to severe psoriasis, including $25 \%$ with PsA, adalimumab treatment resulted in improvements of $11.1 \%$ and $15.5 \%$ in total work productivity impairment and total AI, respectively, compared with placebo [15].

Although the safety and effectiveness of adalimumab in Japanese patients with psoriasis 
has been demonstrated in a postmarketing surveillance (PMS) study [16], the impact of treatment with biologics on Work Productivity and Activity Impairment (WPAI) in Japanese patients with PsA has not been reported. Therefore, we conducted this PMS study to investigate the effectiveness of adalimumab treatment in reducing WPAI in patients with PsA in real-world clinical practice in Japan.

\section{METHODS}

\section{Study Design}

This single-arm, multicenter, prospective cohort, PMS study was conducted from December 2014 to March 2017 at 75 centers in Japan. Patients were registered from December 2014 to September 2016 and were followed up for 24 weeks. Patient assessment data were collected from case report forms at the study centers. This study was conducted in compliance with Good Postmarketing Study Practice (GPSP) in Japan and was registered at ClinicalTrials.gov (NCT02414633). The study protocol was reviewed and approved in advance by the Pharmaceuticals and Medical Devices Agency of Japan. As per GPSP regulations, institutional review board approval and written informed consent from patients were not required.

\section{Patients}

This study included adalimumab-naïve patients meeting the ClASsification criteria for Psoriatic ARthritis (CASPAR) [17] who were paid workers (including part-time). Bedridden and hospitalized patients with decreased activities of daily living and patients in whom adalimumab was contraindicated were excluded from the study.

\section{Study Endpoints}

The primary endpoint was improvement in OWI score from baseline to week 24 of treatment. Secondary endpoints included changes in WPAI-PsA score (OWI, absenteeism, presenteeism, AI), PsA screening and evaluation (PASE) scores, Disease Activity Scores in 28 joints (DAS28) using C-reactive protein (DAS28[CRP]) and erythrocyte sedimentation rate (DAS28[ESR]), tender joint count (TJC; 68 joints), swollen joint count (SJC; 66 joints), PASI score and response rates (PASI75 and PASI90), patient global assessment (PGA), physician global assessment (PhGA), and pain visual analog scale (VAS) at baseline, weeks 4, 12, 16, and 24; change in American College of Rheumatology (ACR) 20/50/70 rates, Bath Ankylosing Spondylitis Disease Activity Index (BASDAI) score, and Health Assessment QuestionnaireDisability Index (HAQ-DI) score at baseline, week 12, and 24; and presence or absence of spondylitis, dactylitis, enthesitis, and nail psoriasis at baseline and last evaluation. Safety was evaluated using the incidence of adverse events (AEs) and serious AEs. In addition, baseline factors impacting OWI were analyzed, and correlation between changes from baseline to last evaluation in OWI scores and effectiveness scores were assessed.

\section{Study Measures}

Scores of the WPAI-PsA were assessed using a questionnaire adapted from the WPAI:PsA V2.0 [18] that comprises the following questions: Q1-Currently employed or not; Q2-Number of hours of work missed due to PsA; Q3Number of hours of work missed due to other reasons; Q4-Number of actual hours worked; Q5-Effect of PsA in reducing productivity during work on a scale of 0 (no effect) to 10 (substantial effect); and Q6-Effect of PsA on regular daily activities on a scale of 0 (no effect) to 10 (substantial effect). OWI, absenteeism, presenteeism, and AI scores were calculated as a percentage of $[\mathrm{Q} 2 /(\mathrm{Q} 2+\mathrm{Q} 4)]+[(1-\mathrm{Q} 2 /$ $(\mathrm{Q} 2+\mathrm{Q} 4)) \times(\mathrm{Q} 5 / 10)], \quad \mathrm{Q} 2 /(\mathrm{Q} 2+\mathrm{Q} 4), \mathrm{Q} 5 / 10$ and $\mathrm{Q} 6 / 10$, respectively, where $\mathrm{Q} 2$ to $\mathrm{Q} 6$ were responses to the respective questions, with the past 7 days as the recall period. Higher scores indicated greater work impairment and lower work productivity.

For assessment details of the other effectiveness measures, including PASE, HAQ-DI, BASDAI, PASI, ACR responses, DAS28(CRP), and 
DAS28(ESR), see the appendix in the electronic supplementary material.

\section{Statistical Analysis}

The target sample size was 130 patients, taking into consideration the number of cases required to detect the same amount of changes in OWI, with a two-sided significance level of $5 \%$ and a detection power of $80 \%$, as observed in the OWI study by Kimball et al. [15], the dropout rate up to 24 weeks in a previous PMS study of psoriasis vulgaris and PsA [16], and the proportion of OWI-evaluable patients (85\%) in a previous PMS study of patients with rheumatoid arthritis (RA) [19].

The safety population included all patients in whom case report forms were completed. Patients who did not receive adalimumab, received adalimumab before signing the contract, were not registered during the registration period, did not meet the enrollment criteria, were duplicate cases, or in whom safety was unevaluable were excluded from the safety population. The effectiveness population comprised all patients from the safety population in whom effectiveness could be evaluated, that is, those patients in whom OWI data at baseline and after administration of adalimumab were available. Categorical data were summarized as number and percentage of cases and quantitative data were summarized as number, percentage, mean, standard deviation (SD), median, and range (minimum, maximum). The descriptive statistics of baseline effectiveness scores and changes from baseline were summarized, and a paired $t$ test was performed to calculate $p$ values. Missing data were not imputed for analysis. The observed effectiveness scores at the last evaluation time point were analyzed for all patients, including those who discontinued early, to adjust for the missing data of patients who discontinued early because of insufficient response to adalimumab treatment. The numbers and percentages of PASI75, PASI90, ACR20, ACR50, and ACR70 responders were summarized, and the 95\% confidence intervals (CIs) were calculated using the Clopper-Pearson method for the response rates.
Correlations between changes from baseline to last evaluation in OWI scores and each effectiveness score were evaluated using a Pearson correlation coefficient, and $p$ values were calculated using the correlation coefficient test. For subgroup analysis of baseline factors affecting OWI, $t$ tests (for factors with two categories) and one-way analysis of variance (for factors with three or more categories) were used. Inferential statistical analyses were performed at a nominal two-sided significance level of 0.05 . Statistical analysis was performed using SAS version 9.3 or higher (SAS Institute, Cary, NC, USA).

Safety events were summarized using the Medical Dictionary for Regulatory Activities version 20.0. Multiple events by preferred term within the same system organ class (SOC) in one patient were counted only once.

\section{RESULTS}

\section{Patient Disposition, Demographics, and Characteristics}

Overall, 148 patients were registered in the study and were included in the safety population. Effectiveness could not be evaluated in 42 patients; therefore, the effectiveness population comprised 106 patients. Among the safety population, 25 patients $(16.9 \%)$ discontinued treatment for at least one reason, including onset of AEs $(n=11)$, ineffectiveness $(n=7)$, patient request $(n=2)$, financial reasons (inability to afford copayment; $n=2$ ), hospital transfer/lost to follow-up $(n=3)$, improvement in symptoms $(n=1)$, and other reasons $(n=1)$.

The effectiveness population comprised mostly men (72.6\%); the mean (SD) age was 49.3 ( \pm 10.7 ) years (Table 1 ). Mean (SD) weight, body mass index (BMI), and duration of skin and joint symptoms were $71.2( \pm 14.5) \mathrm{kg}$ $(n=85), \quad 25.3 \quad( \pm 4.4) \mathrm{kg} / \mathrm{m}^{2} \quad(n=79), \quad 14.8$ $( \pm 12.2)$ years $(n=91)$, and $4.4( \pm 5.1)$ years $(n=94)$, respectively. In 72 patients, $15.0 \%$ $( \pm 18.2 \%)$ of body surface area was affected by psoriasis, mostly plaque-type (85.8\%). Overall, $40.6 \%$ of patients had received methotrexate, $10.4 \%$ had received biologics, and $9.4 \%$ had 
Table 1 Baseline demographics and patient characteristics (effectiveness population)

\begin{tabular}{|c|c|c|}
\hline Characteristic & Number $^{a}$ & Value \\
\hline Age (years) & 106 & $49.3 \pm 10.7$ \\
\hline Sex, male & 106 & $77(72.6)$ \\
\hline Weight $(\mathrm{kg})$ & 85 & $71.2 \pm 14.5$ \\
\hline BMI $\left(\mathrm{kg} / \mathrm{m}^{2}\right)$ & 79 & $25.3 \pm 4.4$ \\
\hline Type of rash & 106 & \\
\hline Plaque & & $91(85.8)$ \\
\hline Erythrodermic & & $7(6.6)$ \\
\hline Pustular & & $4(3.8)$ \\
\hline Guttate & & $1(0.9)$ \\
\hline No rash & & $3(2.8)$ \\
\hline BSA of rash (\%) & 72 & $15.0 \pm 18.2$ \\
\hline Duration of skin symptoms (years) & 91 & $14.8 \pm 12.2$ \\
\hline Duration of joint symptoms (years) & 94 & $4.4 \pm 5.1$ \\
\hline Comorbidities, present & 106 & $46(43.4)$ \\
\hline Past medical history, present & $106^{\mathrm{b}}$ & $20(18.9)$ \\
\hline Smoking history & 106 & \\
\hline Nonsmoker & & $42(39.6)$ \\
\hline Current smoker & & $26(24.5)$ \\
\hline Past smoker & & $15(14.2)$ \\
\hline Unknown & & $23(21.7)$ \\
\hline Previous treatment, present & 106 & $102(96.2)$ \\
\hline Biologics $^{c}$ & & $11(10.4)$ \\
\hline Infliximab & & $7(6.6)$ \\
\hline Ustekinumab & & $3(2.8)$ \\
\hline Other & & $1(0.9)$ \\
\hline Topical $^{\mathrm{c}}$ & & $75(70.8)$ \\
\hline Corticosteroid & & $67(63.2)$ \\
\hline Vitamin $\mathrm{D}_{3}$ derivatives & & $53(50.0)$ \\
\hline Other & & $12(11.3)$ \\
\hline $\mathrm{Oral}^{\mathrm{c}}$ & & $84(79.2)$ \\
\hline NSAID & & $47(44.3)$ \\
\hline Methotrexate & & $43(40.6)$ \\
\hline Corticosteroid & & $18(17.0)$ \\
\hline
\end{tabular}


Table 1 continued

\begin{tabular}{|c|c|c|}
\hline Characteristic & Number $^{a}$ & Value \\
\hline Cyclosporin & & $13(12.3)$ \\
\hline DMARD (except methotrexate) & & $10(9.4)$ \\
\hline Retinoid & & $7(6.6)$ \\
\hline Other & & $11(10.4)$ \\
\hline Concomitant treatment, present & 106 & $90(84.9)$ \\
\hline \multicolumn{3}{|l|}{ Topical $^{\mathrm{c}}$} \\
\hline Corticosteroid & & $30(28.3)$ \\
\hline Vitamin $\mathrm{D}_{3}$ derivatives & & $27(25.5)$ \\
\hline Other & & $14(13.2)$ \\
\hline \multicolumn{3}{|l|}{ Oral $^{c}$} \\
\hline Methotrexate & & $38(35.8)$ \\
\hline NSAID & & $28(26.4)$ \\
\hline Corticosteroid & & $13(12.3)$ \\
\hline Cyclosporin & & $0(0.0)$ \\
\hline DMARD (except methotrexate) & & $5(4.7)$ \\
\hline Retinoid & & $2(1.9)$ \\
\hline Other & & $55(51.9)$ \\
\hline
\end{tabular}

Results are presented as the mean \pm standard deviation or $n(\%)$

$B M I$ body mass index, BSA body surface area, DMARD disease-modifying antirheumatic drug, NSAID nonsteroidal antiinflammatory drug

a Number of cases excluding unknown or cases with missing values

b Past medical history was unknown in one patient

c Data are not mutually exclusive because at least one treatment was possible in a single patient

received a disease-modifying antirheumatic drug (DMARD) other than methotrexate. The baseline demographics and characteristics of the 42 patients who were excluded from the effectiveness population were similar to that of those who were included.

\section{Treatment Duration and Dosage}

The safety population received $11.6( \pm 2.9)$ adalimumab injections throughout 24 weeks.
Among those who received an initial dose of $80 \mathrm{mg}, 15$ and 54 patients continued with 80and 40-mg q2w regimens, respectively, and 10 patients had dose escalation to $80 \mathrm{mg}$ after receiving $40 \mathrm{mg}$ as the second dose. The initial and maintenance dosage in 22 patients was $40 \mathrm{mg}$ q2w; most of these patients (77.3\%) were receiving concomitant methotrexate. Other dosing regimens were used in the five remaining patients. Patients who escalated to the 80-mg dose due to inadequate response had higher baseline body weight $(86.4[ \pm 17.1] \mathrm{kg})$, 
BMI $(30.3[ \pm 5.7]) \mathrm{kg} / \mathrm{m}^{2}$, and VAS scores (PGA, $76.3[ \pm 34.4] ;$ PhGA, $49.3[ \pm 41.1]$, pain, 68.5 $[ \pm 41.3])$ than those receiving the other dosing regimens.

\section{Effectiveness}

Adalimumab treatment significantly improved OWI scores from baseline $(40.2 \pm 32.8 ; n=106)$ to week $24(16.3 \pm 23.9 ; n=79 ;$ difference, $-25.2 \pm 35.3 ; p<0.0001 ;$ Fig. 1$)$. Significant improvement $(p<0.05)$ in all WPAI-PsA domain scores was observed as early as 4 weeks after starting treatment with adalimumab (Fig. 1). Presenteeism scores $(37.5[ \pm 32.0]$ to $14.8[ \pm 21.0] ;$ difference, $-24.3 \quad[ \pm 33.4]$; $p<0.0001)$ and $\mathrm{AI}(41.7[ \pm 30.1]$ to 14.3 $[ \pm 19.0]$; difference, $-27.1 \quad[ \pm 32.7]$; $p<0.0001)$ scores improved significantly and consistently from baseline to week 24 . Although the absenteeism score did not improve consistently over time, a significant improvement
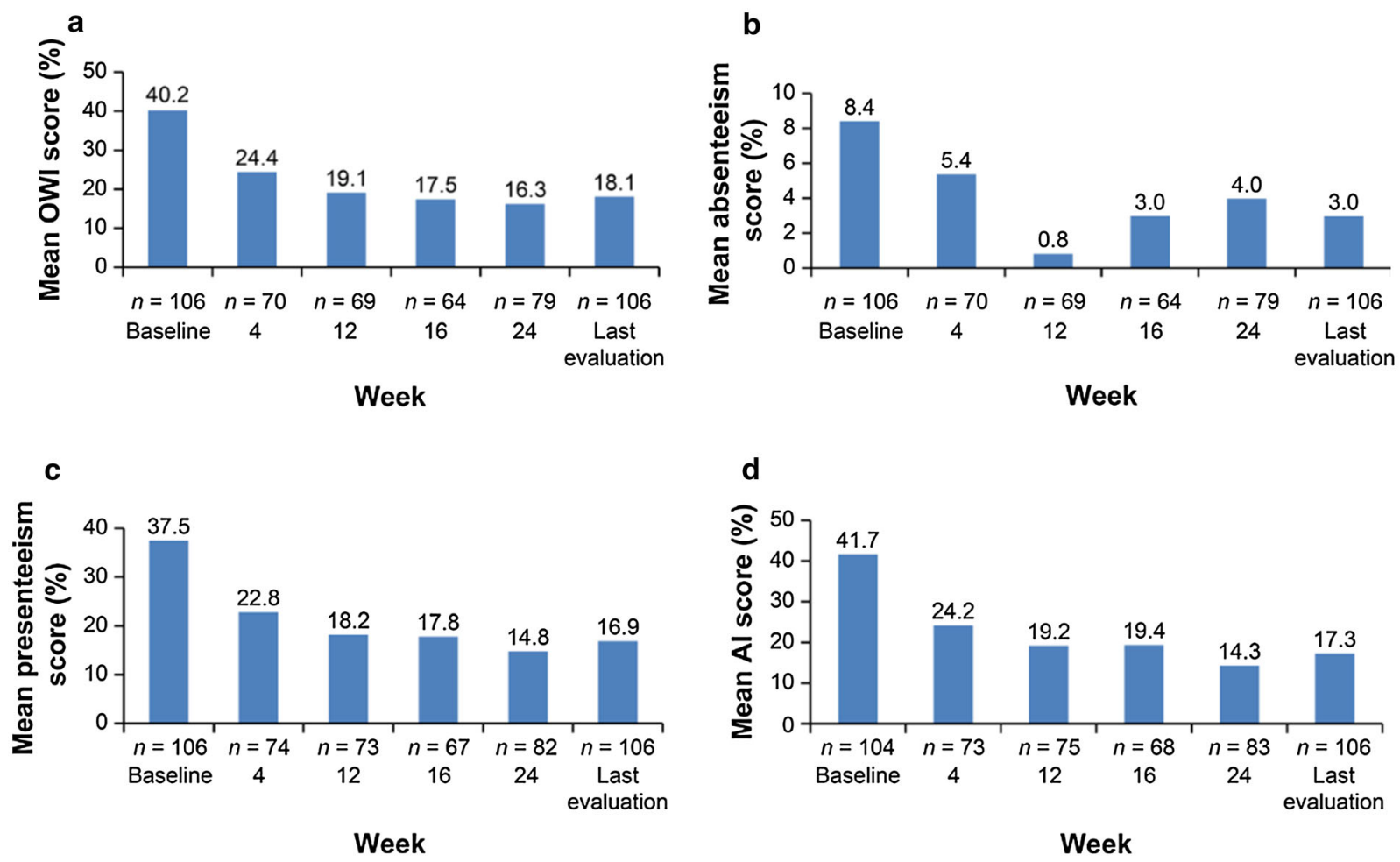

\begin{tabular}{|l|c|c|c|}
\hline $\mathbf{e}$ & \multicolumn{2}{|c|}{ Change in score, mean \pm SD } \\
\hline & 16 weeks & 24 weeks & At last evaluation time point \\
\hline OWI, \% & $-25.9 \pm 32.3^{\star}$ & $-25.2 \pm 35.3^{\star}$ & $-22.2 \pm 35.5^{\star}$ \\
\hline Absenteeism, \% & $-5.4 \pm 17.5^{\star *}$ & $-4.9 \pm 20.1^{\star *}$ & $-5.5 \pm 20.7^{\star \star}$ \\
\hline Presenteeism, \% & $-24.9 \pm 31.5^{\star}$ & $-24.3 \pm 33.4^{\star}$ & $-20.6 \pm 34.0^{\star}$ \\
\hline Al, \% & $-25.0 \pm 32.0^{\star}$ & $-27.1 \pm 32.7^{\star}$ & $-24.1 \pm 32.7^{\star}$ \\
\hline
\end{tabular}

Fig. 1 Change in WPAI domain scores: a overall work impairment; $\mathbf{b}$ absenteeism; c presenteeism; $\mathbf{d} \mathrm{AI}$; e change at 16 weeks, 24 weeks, and last evaluation time point. ${ }^{a}$ Median (range) scores at baseline and 24 weeks are presented in Table S2. ${ }^{*} p<0.0001 ;{ }^{* *} p<0.05 . A I$ activity impairment, $S D$ standard deviation, $O W I$ overall work impairment, WPAI Work Productivity and Activity Impairment 
$(-4.9[ \pm 20.1] ; p=0.0334)$ was obtained at week 24 .

After 24 weeks of adalimumab treatment, PASI75 and PASI90 rates (95\% CI) were 63\% (51.5-73.4) and 42\% (31.1-53.5), respectively, and were 58\% (47.7-67.8) and 39\% (29.4-49.3) at the last evaluation (Fig. 2a). ACR20, ACR50, and ACR70 response rates $(95 \% \mathrm{CI})$ were $91.3 \%$ (79.2-97.6), $76.1 \%(61.2-87.4)$, and $58.7 \%$ (43.2-73.0), respectively, after 24 weeks of adalimumab treatment, and $86.2 \%$ (74.6-93.9), $70.7 \%$ (57.3-81.9), and 53.4\% (39.9-66.7) at the last evaluation (Fig. 2b). Joint symptoms, skin symptoms, disease activity associated with PsA/ spondyloarthritis, and functional parameters significantly improved $(p<0.0001)$ over the study period, as indicated by consistent improvements in corresponding effectiveness endpoints from baseline to week 24 or the last evaluation (Table 2).

Among patients with enthesitis (36.8\%), dactylitis (55.7\%), spondylitis (29.2\%), and nail psoriasis $(50.9 \%)$ at baseline, symptoms resolved in $53.8 \%, 61.0 \%, 67.7 \%$, and $20.4 \%$, respectively, by the last evaluation (Table 3 ). Among patients without these symptoms at baseline, one each developed enthesitis (1.6\%), dactylitis (2.4\%), and nail psoriasis $(2.3 \%)$, and none developed spondylitis by the last evaluation.

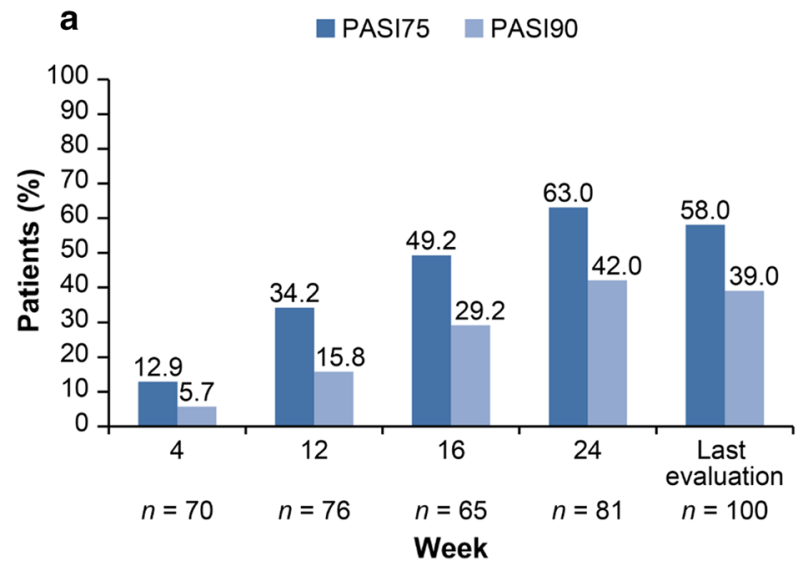

\section{Factors Affecting OWI Improvement}

Past medical history; previous oral NSAID use; and PASE, DAS28(CRP), HAQ-DI, and BASDAI scores at baseline significantly affected improvement in OWI scores from baseline to the last evaluation. Patients with previous oral NSAID use and higher PASE, DAS28(CRP), HAQDI, and BASDAI scores had higher OWI scores at baseline and showed greater improvement in OWI at the last evaluation (Table 3). The improvements in OWI scores from baseline to the last evaluation for all baseline factors are shown in Table S1. A significant correlation was observed between improvement from baseline to the last evaluation in PASE $(r=0.6284$; $p<0.0001$; Fig. S1a), DAS28(CRP) $(r=0.6059$; $p<0.0001 ; \quad$ Fig. S1b), BASDAI $\quad(r=0.7281$; $p<0.0001$; Fig. S1c), and HAQ-DI $(r=0.6161$; $p<0.0001$; Fig. S1d) scores, and OWI scores. A weak correlation was seen between improvement from baseline to the last evaluation in TJC $(r=0.3371 ; p=0.0007)$ and SJC $(r=0.2847$; $p=0.0049)$ and OWI scores, and no correlation was noted between improvement in PASI $(r=0.0622 ; p=0.5493)$ and OWI scores.

\section{Safety}

Overall, 39 AEs were reported in 32 patients (21.6\%), with four serious AEs in four patients (2.7\%). The SOCs with the highest incidences

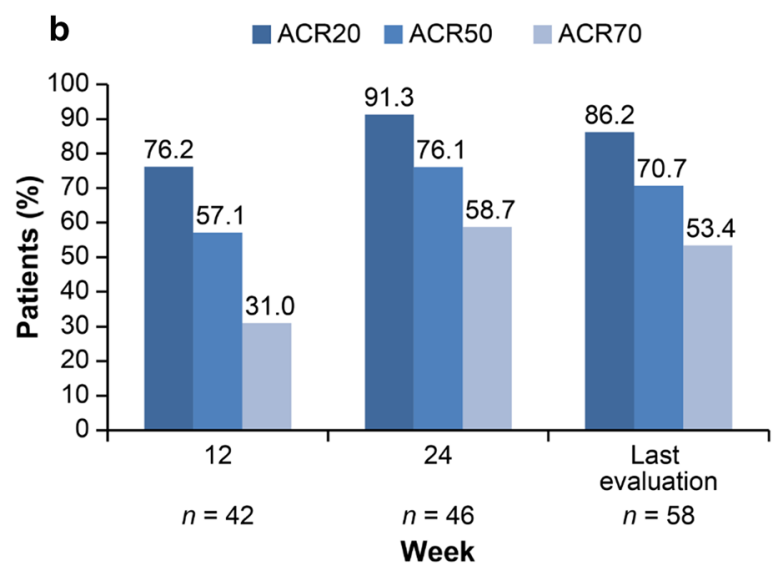

Fig. 2 Change in rate of skin symptoms and joint symptoms over the study: a skin symptoms (PASI); $\mathbf{b}$ joint symptoms (ACR). ACR American College of Rheumatology, PASI Psoriasis Area and Severity Index 


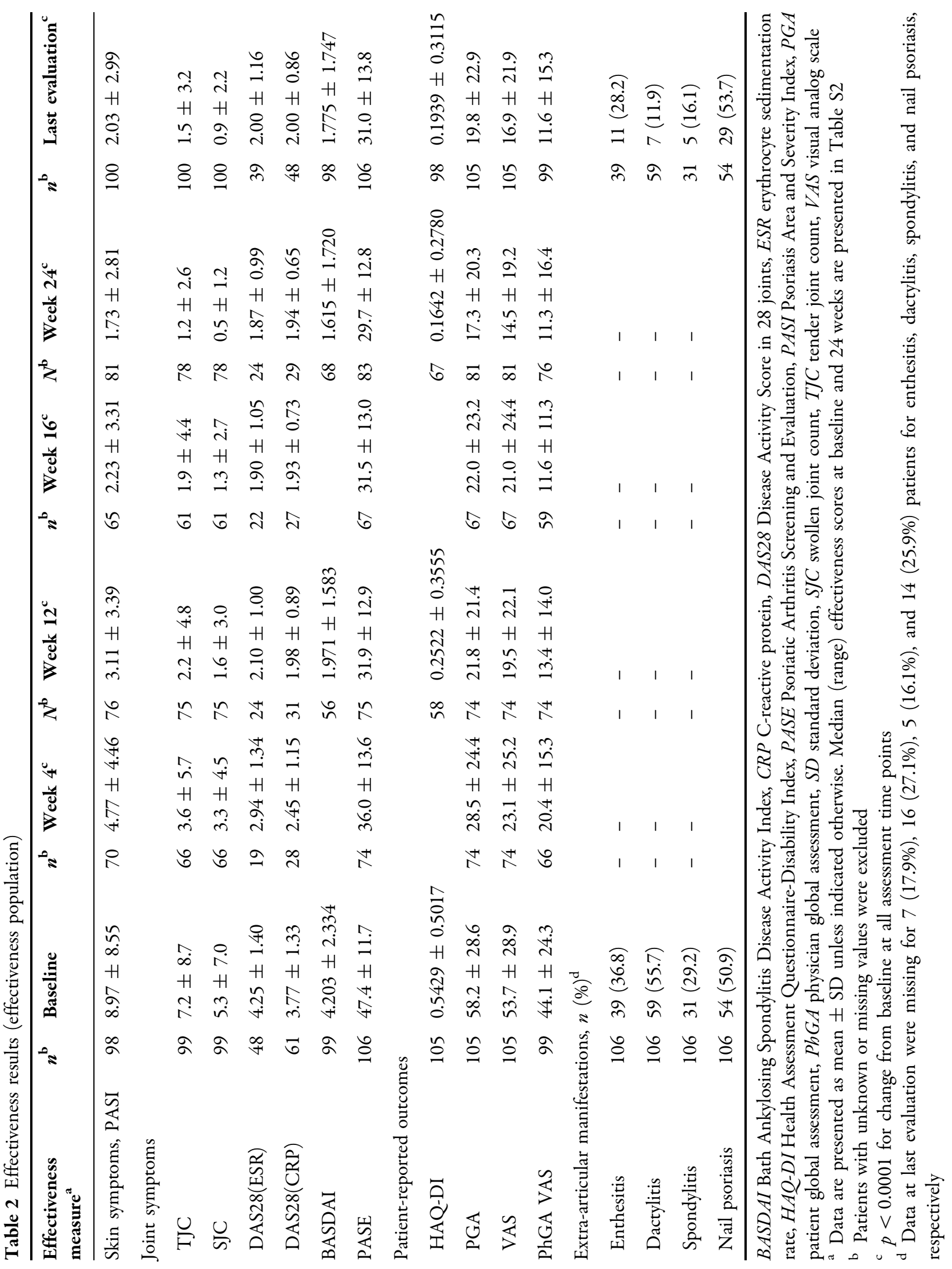


Table 3 Factors affecting OWI score (effectiveness population)

\begin{tabular}{|c|c|c|c|c|c|}
\hline Baseline factor ${ }^{\mathrm{a}, \mathrm{b}}$ & $n^{\mathrm{c}}$ & Baseline & Last evaluation & Change in score & $p$ value $^{\mathrm{d}}$ \\
\hline PASE & & & & & $0.0098^{\mathrm{e}}$ \\
\hline$\geq 47$ & 63 & $54.60 \pm 29.66$ & $25.13 \pm 28.12$ & $-29.47 \pm 36.78$ & \\
\hline$<47$ & 43 & $19.16 \pm 25.18$ & $7.67 \pm 17.02$ & $-11.49 \pm 30.95$ & \\
\hline DAS28(CRP) & & & & & $0.0026^{\mathrm{f}}$ \\
\hline$>5.1$ & 10 & $75.92 \pm 22.67$ & $24.00 \pm 30.98$ & $-51.92 \pm 32.89$ & \\
\hline$>3.2$ to $\leq 5.1$ & 32 & $50.49 \pm 29.42$ & $17.99 \pm 20.41$ & $-32.50 \pm 35.39$ & \\
\hline$>2.6$ to $\leq 3.2$ & 8 & $15.00 \pm 14.14$ & $16.25 \pm 27.74$ & $1.25 \pm 26.42$ & \\
\hline$\leq 2.6$ & 11 & $15.45 \pm 29.79$ & $8.18 \pm 12.50$ & $-7.27 \pm 31.33$ & \\
\hline Unknown/missing & 45 & $35.53 \pm 31.11$ & $19.51 \pm 29.75$ & $-16.02 \pm 33.14$ & \\
\hline HAQ-DI & & & & & $0.0004^{\mathrm{f}}$ \\
\hline$>1.5$ & 4 & $86.43 \pm 9.44$ & $27.50 \pm 17.08$ & $-58.93 \pm 25.35$ & \\
\hline$>1.0$ to $\leq 1.5$ & 12 & $75.42 \pm 21.47$ & $28.33 \pm 32.15$ & $-47.08 \pm 35.06$ & \\
\hline$>0.5$ to $\leq 1.0$ & 29 & $53.21 \pm 31.09$ & $23.45 \pm 28.19$ & $-29.76 \pm 36.93$ & \\
\hline$\leq 0.5$ & 60 & $24.33 \pm 24.87$ & $13.06 \pm 22.62$ & $-11.28 \pm 31.07$ & \\
\hline Unknown/missing & 1 & 10.00 & 0.00 & -10.00 & \\
\hline BASDAI & & & & & $<0.0001^{\mathrm{e}}$ \\
\hline$\geq 4$ & 48 & $61.86 \pm 28.05$ & $25.70 \pm 30.19$ & $-36.16 \pm 37.70$ & \\
\hline$<4$ & 51 & $19.42 \pm 21.76$ & $10.78 \pm 16.83$ & $-8.63 \pm 22.73$ & \\
\hline Unknown/missing & 7 & $43.47 \pm 36.50$ & $18.57 \pm 32.88$ & $-24.90 \pm 59.13$ & \\
\hline Previous oral NSAID & & & & & $0.0142^{\mathrm{e}}$ \\
\hline Absent & 59 & $31.08 \pm 30.15$ & $16.40 \pm 25.01$ & $-14.67 \pm 33.47$ & \\
\hline Present & 47 & $51.70 \pm 32.77$ & $20.12 \pm 26.52$ & $-31.58 \pm 36.10$ & \\
\hline Past medical history & & & & & $0.0455^{\mathrm{e}}$ \\
\hline Absent & 85 & $43.13 \pm 33.51$ & $17.13 \pm 24.92$ & $-26.00 \pm 34.07$ & \\
\hline Present & 20 & $29.39 \pm 28.05$ & $20.89 \pm 29.13$ & $-8.50 \pm 37.82$ & \\
\hline Unknown & 1 & 10.00 & 40.00 & 30.00 & \\
\hline
\end{tabular}

BASDAI Bath Ankylosing Spondylitis Disease Activity Index, CRP C-reactive protein, DAS28 Disease Activity Score in 28 joints, HAQ-DI Health Assessment Questionnaire-Disability Index, NSAID nonsteroidal anti-inflammatory drug, OWI overall work impairment, PASE Psoriatic Arthritis Screening and Evaluation, $S D$ standard deviation

a All values are mean $\pm S D$

b Patients with values missing before treatment initiation (between 28 days prior to and the first administration day of adalimumab) were classified as "unknown/missing”

c Patients with missing values were excluded from this analysis

$\mathrm{d}$ The significance test was performed on the score before administration and the amount of change at final evaluation

${ }^{\mathrm{c}} p$ values were assessed using the $t$ test

$\mathrm{f} p$ values were assessed using a one-way analysis of variance

were infections and infestations (8.1\%), followed by respiratory, thoracic, and mediastinal disorders (3.4\%), and general disorders and administration site conditions, and blood investigations (2.7\% each; Table 4$)$.

\section{DISCUSSION}

The effect of adalimumab on RA-related WPAI and the association between changes in disease activity and WPAI outcomes in routine rheumatology practice in Japan were first evaluated in the ANOUVEAU study [19]. The current PMS study is the first to evaluate the effectiveness of a biologic (adalimumab) in improving WPAI using a validated Japanese version of the WPAI instrument in Japanese patients with PsA. Results from this PMS study indicated that adalimumab treatment is effective in improving PsA-related WPAI and overall disease activity in Japanese patients with PsA 
Table 4 Safety profile (safety population)

\begin{tabular}{|c|c|c|}
\hline$n=148$ & Total AEs & Serious AEs \\
\hline Overall, $n(\%)$ & $32(21.6)$ & $4(2.7)$ \\
\hline Number of events & 39 & 4 \\
\hline \multicolumn{3}{|l|}{ AEs by SOC and $\mathrm{PT}^{\mathrm{a}}, n(\%)$} \\
\hline Infections and infestations & $12(8.1)$ & $1(0.7)$ \\
\hline Viral URTI & $3(2.0)$ & 0 \\
\hline Staphylococcal cellulitis & $1(0.7)$ & $1(0.7)$ \\
\hline Bronchiolitis & $1(0.7)$ & 0 \\
\hline Bronchitis & $1(0.7)$ & 0 \\
\hline Cellulitis & $1(0.7)$ & 0 \\
\hline Paronychia & $1(0.7)$ & 0 \\
\hline Pharyngitis & $1(0.7)$ & 0 \\
\hline Sinusitis & $1(0.7)$ & 0 \\
\hline Viral infection & $1(0.7)$ & 0 \\
\hline Herpes zoster infection neurological & $1(0.7)$ & 0 \\
\hline Respiratory, thoracic, and mediastinal disorders & $5(3.4)$ & $1(0.7)$ \\
\hline Interstitial lung disease & $1(0.7)$ & $1(0.7)$ \\
\hline Asthma & $1(0.7)$ & 0 \\
\hline Cough & $1(0.7)$ & 0 \\
\hline Upper respiratory tract inflammation & $1(0.7)$ & 0 \\
\hline Pulmonary mass & $1(0.7)$ & 0 \\
\hline Blood investigations & $4(2.7)$ & 0 \\
\hline Alanine aminotransferase increased & $1(0.7)$ & 0 \\
\hline Aspartate aminotransferase increased & $1(0.7)$ & 0 \\
\hline Transaminases increased & $1(0.7)$ & 0 \\
\hline Antinuclear antibody positive & $1(0.7)$ & 0 \\
\hline Hepatic enzyme increased & $1(0.7)$ & 0 \\
\hline Cell marker increased & $1(0.7)$ & 0 \\
\hline General disorders and administration site conditions & $4(2.7)$ & $1(0.7)$ \\
\hline Paradoxical drug reaction & $2(1.4)$ & $1(0.7)$ \\
\hline Injection site erythema & $1(0.7)$ & 0 \\
\hline Injection site pruritus & $1(0.7)$ & 0 \\
\hline Edema & $1(0.7)$ & 0 \\
\hline
\end{tabular}


Table 4 continued

\begin{tabular}{|c|c|c|}
\hline$n=148$ & Total AEs & Serious AEs \\
\hline Hepatobiliary disorders & $3(2.0)$ & 0 \\
\hline Hepatic function abnormal & $2(1.4)$ & 0 \\
\hline Liver disorder & $1(0.7)$ & 0 \\
\hline Skin and subcutaneous tissue disorders & $3(2.0)$ & 0 \\
\hline Eczema & $1(0.7)$ & 0 \\
\hline Rash & $1(0.7)$ & 0 \\
\hline Skin symptoms & $1(0.7)$ & 0 \\
\hline Musculoskeletal and connective tissue disorders & $2(1.4)$ & 0 \\
\hline Arthralgia & $1(0.7)$ & 0 \\
\hline Psoriatic arthropathy & $1(0.7)$ & 0 \\
\hline Renal and urinary disorders & $1(0.7)$ & $1(0.7)$ \\
\hline Lupus nephritis & $1(0.7)$ & $1(0.7)$ \\
\hline Nervous system disorders & $1(0.7)$ & 0 \\
\hline Hypoesthesia & $1(0.7)$ & 0 \\
\hline Gastrointestinal disorders & $1(0.7)$ & 0 \\
\hline Oral disorders & $1(0.7)$ & 0 \\
\hline
\end{tabular}

$A E$ adverse event, $P T$ preferred term, SOC system organ class, URTI upper respiratory tract infection

${ }^{a}$ Multiple events by PT in one patient were counted once in the corresponding SOC

over a 24-week observation period. There were consistent improvements in OWI, presenteeism, and AI over time with adalimumab treatment. Although absenteeism also improved significantly by study end, the improvement was not consistent over time, perhaps because only a few patients had a score greater than 0 before treatment initiation, leading to large variations at each time point during the study. Adalimumab treatment also led to significant improvement in joint, skin, and spinal symptoms and functional impairment associated with PsA. However, improvement in joint symptoms largely contributed to the improvement of OWI, and patients presenting with higher disease activity related to joint symptoms at baseline showed greater improvement in OWI at the final evaluation. In contrast, no significant association was observed between skin symptoms and OWI. Additionally, significant improvement in OWI with adalimumab treatment was observed regardless of sex, disease duration, or the presence of enthesitis, dactylitis, or spondylitis, suggesting that treatment directed at alleviating disease activity related to joints may lead to improvement of work productivity in patients with PsA.

Improvement in OWI was significantly impacted by past medical history; previous oral NSAID use; and PASE, DAS28(CRP), BASDAI, and HAQ-DI scores. Patients with moderate or high disease activity (DAS28[CRP] > 3.2) and higher PASE, HAQ-DI, and BASDAI scores at baseline, suggestive of more severe disease, had higher baseline OWI scores and showed greater improvement in work productivity. Similarly, patients with previous use of NSAIDs compared 
with those without, likely due to more severe pain, had higher baseline OWI scores and showed greater improvement in work productivity.

Most of the demographic and clinical characteristics of the study population (e.g., a higher percentage of men, mean age in the late $40 \mathrm{~s}$, mean duration of skin symptoms approximately 10 years longer than that of joint symptoms, predominance of plaque-type psoriasis, and almost $25 \%$ of patients who were smokers) were consistent with those observed in a recent survey by the Japanese Society for Psoriasis Research [8]. As in a previous Japanese PMS study (SALSA) [16], where the safety and effectiveness of adalimumab in treating psoriasis was evaluated over 24 weeks, more than $90 \%$ of patients with PsA in our study had received previous treatment, mainly methotrexate, despite its status as an off-label treatment in Japan [11], and had received corticosteroid treatment, mainly in a topical form. However, the percentage of previous oral corticosteroid and methotrexate users was higher in the current PMS study than in the prior SALSA study. Compared with the SALSA study, fewer dermatology departments participated as study centers because arthritis symptoms were evaluated mainly by rheumatologists or orthopedic surgeons in this study. The differences between the treatment perspectives of dermatologists and rheumatologists/orthopedic surgeons could have been attributed to the variation in the percentage of corticosteroid and methotrexate users between the two studies.

The dosage of adalimumab varied in the present PMS study, with approximately $50 \%$ of patients receiving the recommended dosage. Most patients who initiated treatment and continued with a lower dose (40 mg) were using methotrexate, which is not surprising, given the potentially enhanced effectiveness of concomitant treatment. However, methotrexate is not approved for the treatment of patients with PsA in Japan [11]. Patients with escalation to the 80-mg adalimumab dose because of inadequate response to the 40-mg dose weighed more and had higher BMI or poorer VAS scores at baseline than those on other dosing regimens. The strong association between obesity and the severity of psoriasis has been described previously [20]. Moreover, in a subgroup analysis of the phase 3 REVEAL study of patients with moderate to severe psoriasis receiving adalimumab, treatment response was moderately lower with $40 \mathrm{mg}$ q2w dosing in obese than in non-obese patients [21]. Similarly, patients with a dose escalation had a higher BMI than those without dose escalation and exhibited an improved treatment response following dose escalation in a long-term phase $2 / 3$ trial of adalimumab in Japanese patients with moderate to severe plaque psoriasis [22]. Taken together, these results suggest that higher adalimumab doses should be considered in obese patients and those with severe disease. Furthermore, as observed in an earlier study, weight-control interventions in obese patients receiving treatment with a TNF inhibitor could potentially improve treatment response [23].

WPAI is a validated tool and has been used in other studies as well to determine the impact of biologic therapy in improving work disability. Similar to effectiveness results from this study (median [range] scores shown in Table S2), a UK study [24] reported improvement in median productivity loss (OWI; $50 \%$ to $10 \%$ ), presenteeism (40\% to $10 \%)$, and $\mathrm{AI}(60 \%$ to $20 \%)$ scores in patients with active PsA following 6-month treatment with TNF inhibitors in a real-world setting; improvement in patient-reported outcomes was also reported. However, only about $57 \%$ of patients were employed in the UK study, unlike in the present study, which included only patients who were employed. A central-eastern Europe study also reported improvements in mean WPAI scores (OWI, 59.9\% to 22.1\%; presenteeism, $56.7 \%$ to $20.1 \%$ ) after 1 year of treatment with adalimumab in patients with ankylosing spondylitis (AS) and PsA (approx. 27\% of the study population) [25]. Besides differences in the studied populations, the baseline scores were higher in the above studies $[24,25]$ compared with this study. Moreover, $65.8 \%$ of patients with AS compared with $25.8 \%$ of those with PsA were using NSAIDs in the European study [25], indicating more severe pain and potentially greater work impairment in patients with AS than in those with PsA. As a result of these differences, 
it is challenging to compare the WPAI outcomes from the current study with those of other realworld studies. Treatment with other biologics (ustekinumab, apremilast, certolizumab pegol, golimumab, and infliximab) has also been reported to improve self-reported work productivity in patients with PsA in a systematic metaanalysis of five randomized controlled trials. However, randomized controlled settings and variations in WPAI measures used in the five trials that were analyzed preclude any comparison with the current study results [26].

Overall, approximately one-half and twothirds of patients achieved PASI75 at weeks 16 and 24 , respectively, and approximately onethird and two-fifths of patients achieved PASI90 at weeks 16 and 24, respectively, in this study. PASI achievement rates are similar to those reported in the aforementioned prior Japanese PMS study [16]. The changes from baseline to week 24 in DAS28(ESR), DAS28(CRP), and VAS pain scores were also comparable between these studies. The PASI90 rate at 24 weeks (42\%) in this study was the same as that reported in the Adalimumab Effectiveness in Psoriatic Arthritis Trial (ADEPT), comprising a primarily white cohort [27]. However, ACR20, ACR50, and ACR70 response rates obtained at weeks 12 (76.2\%, 57.1\%, and $31.0 \%$, respectively) and 24 $(91.3 \%, 76.1 \%$, and $58.7 \%$, respectively) in this study were numerically higher than those in ADEPT $(58 \%, 36 \%$, and 20\%; and 57\%, 39\%, and $23 \%$, respectively) [27], suggesting comparable effectiveness of adalimumab treatment in improving skin symptoms in white and Japanese patients, with potentially a better response for joint symptoms in Japanese patients. However, the difference could also be attributed to differences in study design and setting.

Multivariate regression analysis in a US study showed that patients with more severe PsA had increased odds of high WPAI domain scores, with the exception of absenteeism [28]. In the REVEAL study, all WPAI domain scores were significantly correlated with disease severity assessed using PASI, PhGA, and the Dermatology Life Quality Index [15]. In contrast, PASI scores were not significantly correlated with OWI in the current PMS study. However, other disease severity measures, particularly those related to joint symptoms such as PASE, DAS28(CRP), BASDAI, and HAQ-DI, were significantly correlated with OWI. These findings are supported by results from a large, multicenter UK study in which work disability was significantly associated with global, physical function, and joint-specific disease, assessed using global and joint activity measures (VAS and HAQ-DI), but not with skin-specific disease [29].

The incidence of AEs and serious AEs was comparable with that in the previous real-world study of patients with psoriasis in Japan [16], and no new or unexpected safety signals were observed.

This study had several strengths. First, the demographics and characteristics of the study population are representative of the real-world psoriasis population in Japan. Second, WPAIPsA was measured from baseline to week 24, providing a glimpse of the long-term impact of adalimumab treatment on WPAI in patients with PsA. This study, however, also had some limitations. Although a central registration system was employed to reduce selection bias, results could still be biased because of the openlabel and observational nature of the study. As a result of the observational design of the study, approximately one-third of enrolled patients had to be excluded from the efficacy analysis set because of unavailability of OWI scores. In addition, routine clinical settings resulted in several limitations, including the inability to control the use of concomitant treatment and the inability to obtain effectiveness scores at all time points.

\section{CONCLUSIONS}

Results from this PMS study suggest that adalimumab treatment improves work productivity and impairment, and reduces disease activity in patients with PsA. Improvement in work impairment was associated with greater jointspecific PsA severity and disability before treatment initiation and improvement in joint symptoms following adalimumab treatment. Finally, no new safety signals were identified 
with adalimumab treatment in this real-world setting.

\section{ACKNOWLEDGMENTS}

We would like to thank the participants of the study.

Funding. AbbVie GK and Eisai Co., Ltd. funded the study, the article processing charges, and the open access fee. AbbVie participated in the collection, analysis, and interpretation of the data, and in the drafting, review, and approval of the manuscript. All authors had full access to all of the data in this study and take complete responsibility for the integrity of the data and accuracy of the data analysis.

Medical Writing, Editorial, and Other Assistance. Medical writing support was provided by Deepali Garg, MBBS, PGDHA, and Maribeth Bogush, PhD, of Cactus Communications and was funded by AbbVie GK and Eisai. Data analysis support was provided by Fujitsu FIP Corporation and was funded by AbbVie GK and Eisai.

Authorship. All named authors meet the International Committee of Medical Journal Editors (ICMJE) criteria for authorship for this article, take responsibility for the integrity of the work as a whole, and have given their approval for this version to be published.

Disclosures. Hidemi Nakagawa is a consultant and/or has received research grants and/or speaker honoraria from Kyowa Hakko Kirin, AbbVie, Eisai, UCB, Mitsubishi Tanabe, Janssen, Eli Lilly, LEO Pharma, Maruho, and MSD. Yoshiya Tanaka has received speakers fees and/ or honoraria from Daiichi Sankyo, Astellas, Eli Lilly, Chugai, Sanofi, AbbVie, YL Biologics, Bristol-Myers Squibb, GlaxoSmithKline, UCB, Mitsubishi Tanabe, Novartis, Eisai, Takeda, Janssen, and Asahi Kasei and has received research grants from Mitsubishi Tanabe, BristolMyers Squibb, Eisai, Chugai, Takeda, AbbVie, Astellas, Daiichi Sankyo, Ono, MSD, and Taisho
Toyama. Akimichi Morita has received research grants, consulting fees, and/or speakers fees from AbbVie, Celgene, Eli Lilly, Eisai, Janssen, Kyowa Hakko Kirin, LEO Pharma, Maruho, Mitsubishi Tanabe, and Novartis. Shigetoshi Sano has received research grants, consulting fees, and/or speakers fees from AbbVie, Eli Lilly, Janssen, Kyowa Hakko Kirin, LEO Pharma, Maruho, Celgene, Mitsubishi Tanabe, and Novartis. Atsuo Taniguchi has received speakers fees from AbbVie, Eisai, Teijin, Chugai, Eli Lilly, Novartis, Janssen, and Mitsubishi Tanabe. Tomoko Kashiwagi is a full-time employee of AbbVie Japan and may own AbbVie stock and options. Junko Kimura is a full-time employee of AbbVie Japan and may own AbbVie stock and options. Takeshi Kawaberi is a full-time employee of AbbVie Japan and may own AbbVie stock and options. Hideto Kameda has received consulting fees, speakers fees, and/or honoraria from AbbVie, Asahi Kasei, Astellas, Bristol-Myers Squibb, Chugai, Eli Lilly, Mitsubishi Tanabe, Novartis, Sanofi, and Janssen, and research grants from AbbVie, Asahi Kasei, Astellas, Chugai, Eisai, Mitsubishi Tanabe, Novartis, Sanofi, and UCB.

Compliance with Ethics Guidelines. This study was conducted in compliance with Good Postmarketing Study Practice (GPSP) in Japan and was registered at ClinicalTrials.gov (NCT02414633). The study protocol was reviewed and approved in advance by the Pharmaceuticals and Medical Devices Agency of Japan. As per GPSP regulations, institutional review board approval and written informed consent from patients were not required.

Prior Presentations at Meetings. Data included in this manuscript were presented at EADV 2018 (Paris, France).

Data Availability. The datasets generated and/or analyzed during the current study are available from the corresponding author on reasonable request.

Open Access. This article is distributed under the terms of the Creative Commons Attribution-NonCommercial 4.0 International 
License (http://creativecommons.org/licenses/ by-nc/4.0/), which permits any noncommercial use, distribution, and reproduction in any medium, provided you give appropriate credit to the original author(s) and the source, provide a link to the Creative Commons license, and indicate if changes were made.

\section{REFERENCES}

1. Armstrong AW, Schupp C, Wu J, Bebo B. Quality of life and work productivity impairment among psoriasis patients: findings from the National Psoriasis Foundation survey data 2003-2011. PLoS One. 2012;7:e52935.

2. Ritchlin CT, Colbert RA, Gladman DD. Psoriatic arthritis. N Engl J Med. 2017;376:2095-6.

3. Tillett W, de-Vries C, McHugh NJ. Work disability in psoriatic arthritis: a systematic review. Rheumatology (Oxford). 2012;51:275-83.

4. Gladman DD. Psoriatic arthritis. Dermatol Ther. 2009;22:40-55.

5. Takahashi H, Nakamura K, Kaneko F, Nakagawa H, Iizuka H, Japanese Society for Psoriasis Research. Analysis of psoriasis patients registered with the Japanese Society for Psoriasis Research from 2002-2008. J Dermatol. 2011;38:1125-9.

6. Yamamoto T, Ohtsuki M, Sano S, et al. Epidemiological analysis of psoriatic arthritis patients in Japan. J Dermatol. 2016;43:1193-6.

7. Yamamoto T, Ohtsuki M, Sano S, et al. Prevalence and current therapies of psoriatic arthritis in Japan: a survey by the Japanese Society of Psoriasis Research in 2016. J Dermatol. 2017;44:e121.

8. Yamamoto T, Kawada A. Clinical characteristics of Japanese patients with psoriatic arthritis: comparison with East Asian countries. J Dermatol. 2018;45:273-8.

9. Hayashi M, Saeki H, Ito T, et al. Impact of disease severity on work productivity and activity impairment in Japanese patients with psoriasis. J Dermatol Sci. 2013;72:188-91.

10. Gossec L, Smolen JS, Ramiro S, et al. European League Against Rheumatism (EULAR) recommendations for the management of psoriatic arthritis with pharmacological therapies: 2015 update. Ann Rheum Dis. 2016;75:499-510.
11. Ohtsuki M, Terui T, Ozawa A, et al. Japanese guidance for use of biologics for psoriasis (the 2013 version). J Dermatol. 2013;40:683-95.

12. Products Approved in FY 2009: New drugs: pharmaceuticals and Medical Devices Agency, Japan. 2009. https://www.pmda.go.jp/files/000153452. pdf. Accessed 6 June 2018.

13. HUMIRA $^{\circledR}$ Package Insert (in Japanese) Pharmaceuticals and Medical Devices Agency, Japan. 2018. http://www.info.pmda.go.jp/downfiles/ph/PDF/ 112130_3999426G2020_1_03.pdf. Accessed 2 Oct 2018.

14. Asahina A, Nakagawa H, Etoh T, Ohtsuki M, Adalimumab MSG. Adalimumab in Japanese patients with moderate to severe chronic plaque psoriasis: efficacy and safety results from a phase II/III randomized controlled study. J Dermatol. 2010;37: 299-310.

15. Kimball AB, Yu AP, Signorovitch J, et al. The effects of adalimumab treatment and psoriasis severity on self-reported work productivity and activity impairment for patients with moderate to severe psoriasis. J Am Acad Dermatol. 2012;66:e67-76.

16. Asahina A, Torii $\mathrm{H}$, Ohtsuki $\mathrm{M}$, et al. Safety and efficacy of adalimumab treatment in Japanese patients with psoriasis: results of SALSA study. J Dermatol. 2016;43:1257-66.

17. Taylor W, Gladman D, Helliwell P, et al. Classification criteria for psoriatic arthritis: development of new criteria from a large international study. Arthritis Rheum. 2006;54:2665-73.

18. WPAI:SHP V2.0: Reilly Associates; updated August 18, 2010. http://www.reillyassociates.net/WPAI_ SHP.html. Accessed 24 Apr 2018.

19. Takeuchi T, Nakajima R, Komatsu S, et al. Impact of adalimumab on work productivity and activity impairment in Japanese patients with rheumatoid arthritis: large-scale, prospective, single-cohort ANOUVEAU study. Adv Ther. 2017;34:686-702.

20. Neimann AL, Shin DB, Wang X, Margolis DJ, Troxel AB, Gelfand JM. Prevalence of cardiovascular risk factors in patients with psoriasis. J Am Acad Dermatol. 2006;55:829-35.

21. Menter A, Gordon KB, Leonardi CL, Gu Y, Goldblum OM. Efficacy and safety of adalimumab across subgroups of patients with moderate to severe psoriasis. J Am Acad Dermatol. 2010;63:448-56.

22. Asahina A, Ohtsuki M, Etoh T, et al. Adalimumab treatment optimization for psoriasis: results of a long-term phase 2/3 Japanese study. J Dermatol. $2015 ; 42: 1042-52$. 
23. Di Minno MN, Peluso R, Iervolino S, et al. Weight loss and achievement of minimal disease activity in patients with psoriatic arthritis starting treatment with tumour necrosis factor $\alpha$ blockers. Ann Rheum Dis. 2014;73:1157-62.

24. Tillett W, Shaddick G, Jobling A, et al. Effect of antiTNF and conventional synthetic disease-modifying anti-rheumatic drug treatment on work disability and clinical outcome in a multicentre observational cohort study of psoriatic arthritis. Rheumatology (Oxford). 2017;56:603-12.

25. Szántó S, Poór G, Opris D, et al. Improved clinical, functional and work outcomes in spondyloarthritides during real-life adalimumab treatment in central-eastern Europe. J Comp Eff Res. 2016;5:475-85.

26. Iragorri N, Hofmeister M, Spackman E, Hazlewood GS. The effect of biologic and targeted synthetic drugs on work- and productivity-related outcomes for patients with psoriatic arthritis: a systematic review. J Rheumatol. 2018;45:1124-30.

27. Mease PJ, Gladman DD, Ritchlin CT, et al. Adalimumab for the treatment of patients with moderately to severely active psoriatic arthritis: results of a double-blind, randomized, placebo-controlled trial. Arthritis Rheum. 2005;52:3279-89.

28. Korman NJ, Zhao Y, Pike J, Roberts J. Relationship between psoriasis severity, clinical symptoms, quality of life and work productivity among patients in the USA. Clin Exp Dermatol. 2016;41:514-21.

29. Tillett W, Shaddick G, Askari A, et al. Factors influencing work disability in psoriatic arthritis: first results from a large UK multicentre study. Rheumatology (Oxford). 2015;54:157-62. 